\title{
Inventaire par télédétection des agro-écosystèmes de bas-fonds dans le centre du Bénin
}

Adéyèmi Chabi ${ }^{1}$

Joseph Oloukoi ${ }^{2}$

Vincent Joseph Mama ${ }^{3}$

Paul Kiepe ${ }^{1}$

${ }^{1}$ Centre du Riz pour I'Afrique

AfricaRice

01 BP 2031

Cotonou

Bénin

<a.chabi@cgiar.org>

$<$ p.kiepe@cgiar.org>

${ }^{2}$ Regional Centre for Training in Aerospace Surveys (RECTAS)

Off Road 1

Obafemi Awolowo University Campus

PMB 5545 Ile-Ife

Nigeria

<oloukoi@rectas.org>

${ }^{3}$ Institut national des recherches agricoles du Bénin

06 BP 1105

Cotonou

Bénin

<mamvincent@yahoo.com>

\begin{abstract}
Résumé
En Afrique subsaharienne, les bas-fonds sont des agro-écosystèmes souvent favorables à la riziculture et aux cultures de contre-saison. Ces ressources potentielles n'ont pas bénéficié d'un développement rapide à cause de leur extrême diversité et du fait de la méconnaissance de leurs potentialités par les acteurs du développement L'objectif central de cette étude est d'estimer l'intérêt d'un système d'information sur les bas-fonds, à partir de l'exemple du secteur de Dassa-Zoumé situé au centre du Bénin. La méthode adoptée pour faire l'inventaire des bas-fonds repose sur le calcul du néocanal NDVI (indice normalisé de végétation) à partir d'une image Landsat de résolution spatiale de $28,5 \mathrm{~m}$, et sur la réalisation de cartes de pente générées à partir du modèle d'élévation de terrain. Cent quatre-vingt-un bas-fonds couvrant une superficie de 1123 hectares ont été identifiés. Environ $60 \%$ des bas-fonds identifiés ont une superficie comprise entre 0 et 5 hectares, tandis que moins de $5 \%$ d'entre eux ont des superficies comprises entre 20 et 25 hectares. L'approche de détection et d'inventaire utilisée dans cette étude, essentiellement fondée sur l'exploitation d'une image satellitale, présente une fiabilité telle qu'elle mérite d'être généralisée pour la recherche des zones basses pour des perspectives agricoles. Elle permet de gagner du temps, comparativement aux autres approches qui nécessitent que tous les bas-fonds soient parcourus et levés sur le terrain.
\end{abstract}

Mots clés : agroécosystème ; bas-fonds ; Bénin ; système d'information géographique ; télédétection.

Thèmes : méthodes et outils ; ressources naturelles et environnement.

\begin{abstract}
Inventory by remote sensing of inland valleys agro-ecosystems in central Benin

In sub-Saharan Africa, inland valleys are types of agro-ecosystems suitable for rice cultivation and anti seasonal crops. These important resources have not been developed enough due to their extreme diversity and to the fact that their potentialities are remain poorly identified by scientists and the actors of development. The present research aimed at estimating the interest of an information system on inland valleys, using the Dassa-Zoumé area in the centre of Benin as the study area. The methodology adopted for the inventory of the inland valleys is based on the calculation of the new channel NDVI (Normalized Difference Vegetation Index) from the Landsat 7-ETM+ image of 2000 with a spatial resolution of $28.5 \mathrm{~m}$ and on the realization of the slope map generated from a DEM (Digital Elevation Model) of the area. A total of 181 inland valleys were identified with a total surface of 1,124 hectares. About $60 \%$ of the valleys identified have an area comprised between 0 and 5 ha while less than 5\% vary from 20 to 25 ha. The detection and inventory approach used in this study, and based essentially on the exploitation of a satellite image, shows a level of reliability that should be extended in the search of lowlands for agricultural perspectives. It facilitates savings in time in comparison to other approaches that would necessitate the surveying of all the inland valleys.
\end{abstract}

Key words: agroecosystems; Benin; geographical information; inland valleys; remote sensing.

Subjects: natural resources and environment; tools and methods.

\footnotetext{
* Pour citer cet article: Chabi A, Oloukoi J, Mama VJ, Kiepe P. Inventaire par télédétection des agro-écosystèmes de bas-fonds dans le centre du Bénin. Cah Agric 2010; 19: 446-53; doi : 10.1684/agr.2010.0434
} 
n Afrique subsaharienne les basfonds présentent un potentiel très important de développement et d'intensification de la production agricole et spécialement rizicole (Raunet, 1985; Mameri et al., 2008). Leur surface est estimée à 85 millions d'hectares, soit $7 \%$ de la superficie totale des terres cultivables, dont seulement 5 à $10 \%$ sont utilisées (Andriesse et Fresco, 1991). Dans les pays en développement comme le Bénin, la mise en valeur des bas-fonds est devenue un enjeu majeur pour le développement agricole. Les communautés villageoises souhaitent en effet développer la riziculture de bas-fonds, qui représente plus de $90 \%$ des surfaces rizicoles du Bénin; mais l'inventaire des bas-fonds reste difficile par manque d'outils adéquats (Danvi et al., 1998). La mise en place d'une base de données spatiales pourrait faciliter l'inventaire de ces ressources.

Dassa-Zoumé fait partie des communes du centre du Bénin, où l'exploitation des bas-fonds est importante, tant pour la riziculture que pour les cultures de contresaison (Mama et al., 2000).

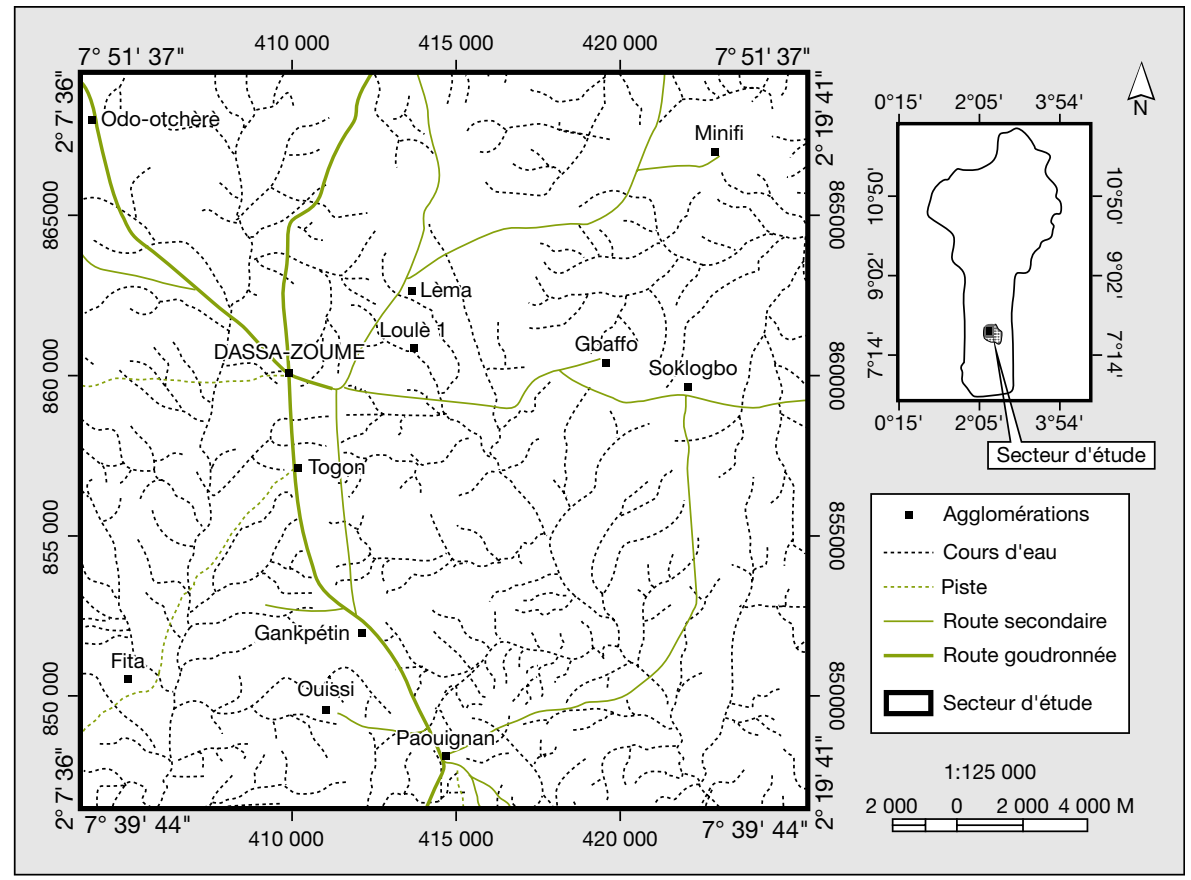

Figure 1. Situation du secteur étudié.

Figure 1. Location of the study area.

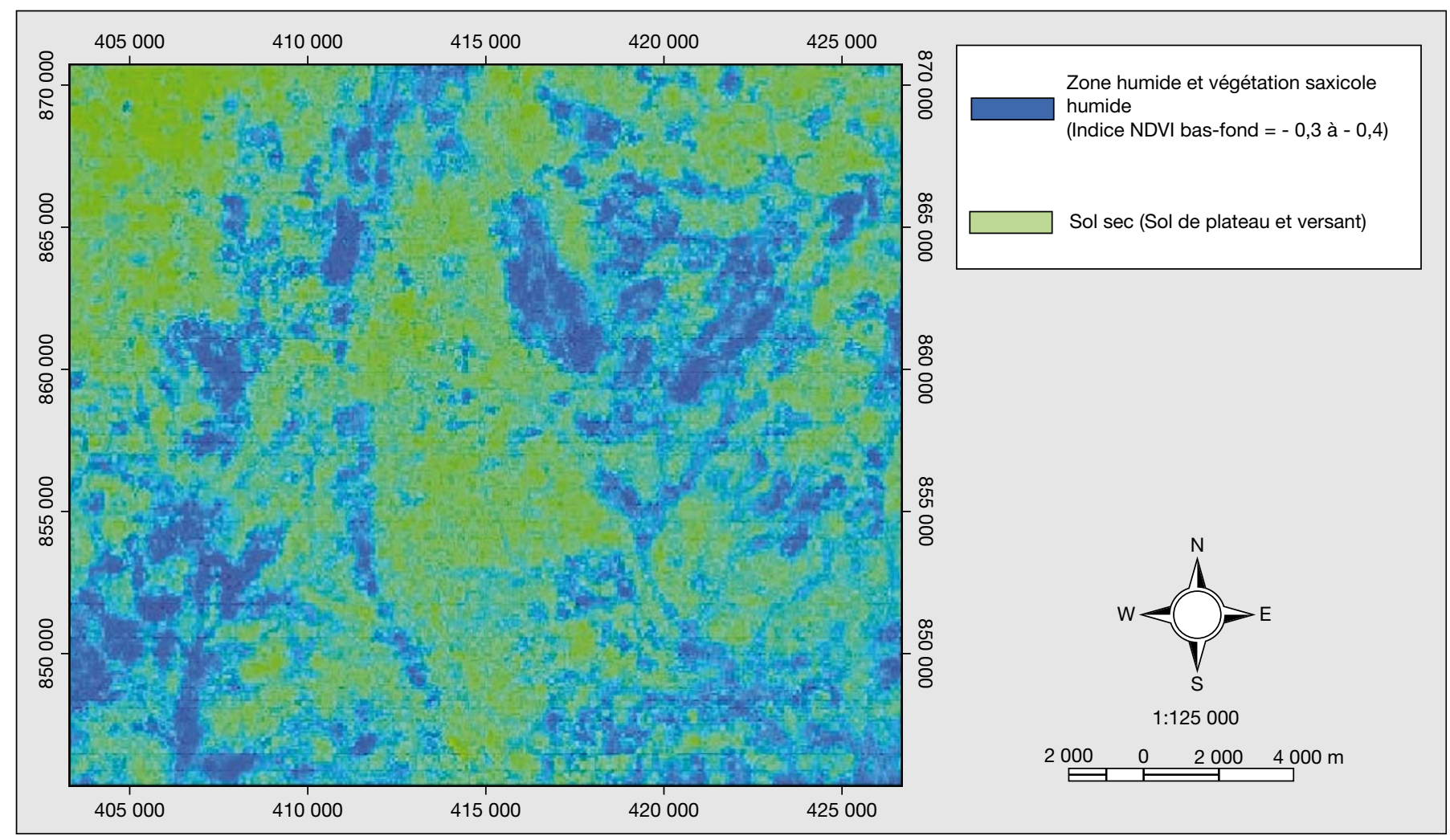

Figure 2. Le néocanal NDVI (indice normalisé de végétation).

Figure 2. NDVI index map. 
L'extrême diversité de ces zones, alliée à l'absence d'une connaissance fiable de leur répartition et de leur potentiel, freine les politiques de développement. Une meilleure connaissance de ces bas-fonds est donc nécessaire (Houndagba et Akouegninou, 1999).

La télédétection peut être d'une grande utilité en facilitant une visualisation de la situation des bas-fonds et l'estimation des surfaces concernées.

La présente étude vise à tester une approche d'inventaire reposant essentiellement sur la télédétection et sur un système d'information géographique (SIG).

\section{Présentation}

\section{de la zone d'étude}

La république du Bénin couvre une superficie d'environ $115800 \mathrm{~km}^{2}$, entre $6^{\circ} 02^{\prime}$ et $12^{\circ} 26^{\prime}$ de latitude Nord et entre $1^{\circ}$ et $3^{\circ} 30^{\prime}$ de longitude Est. La zone d'étude concerne la commune de DassaZoumé située dans le département des Collines (figure 1), situé entre $7^{\circ} 27^{\prime}$ et $8^{\circ} 46^{\prime}$ de latitude Nord et entre $1^{\circ} 39^{\prime}$ et $2^{\circ} 44$ ' de longitude Est. Elle bénéficie d'un climat de transition entre le climat guinéen et le climat soudanien (Afouda, 1990), avec un régime pluviométrique intermédiaire entre la distribution bimodale du Sud et celle unimodale du Nord.

Sur le plan hydrographique, les cours d'eau qui drainent le département des Collines présentent un régime tropical avec une seule période de crue entre août et octobre.

Les collines sont caractérisées par des sols ferrugineux en général, alors que des sols hydromorphes se rencontrent dans les bas-fonds et les dépressions. La végétation naturelle est composée de forêts denses sèches, de forêts claires et de savanes boisées.

La population de la commune de DassaZoumé était, en 2002, de 94000 habitants, soit 55 habitants $/ \mathrm{km}^{2}$ (INSAE, 2003).

\section{Matériel et méthode}

\section{Matériels et données}

Les matériels et données utilisés dans cette étude sont les suivants :

- une image satellite Landsat-7 ETM

+ ortho rectifiée du 13 décembre 2000, avec une résolution de $28,5 \mathrm{~m}$;

- une carte topographique, feuille de Zagnanado au 1/200 000, avec une équidistance de $40 \mathrm{~m}$;

- un GPS (Global Positioning System) pour le relevé des bas-fonds ;

- les logiciels de traitement d'image et SIG Ilwis3.4, Arcview 3.2 et ArcGIS 9.1.

\section{Collecte des données}

Les données primaires utilisées sont l'image Landsat ETM + de décembre 2000 et les données de terrain relevées avec le GPS. Les données secondaires utilisées sont la carte topographique de l'Afrique

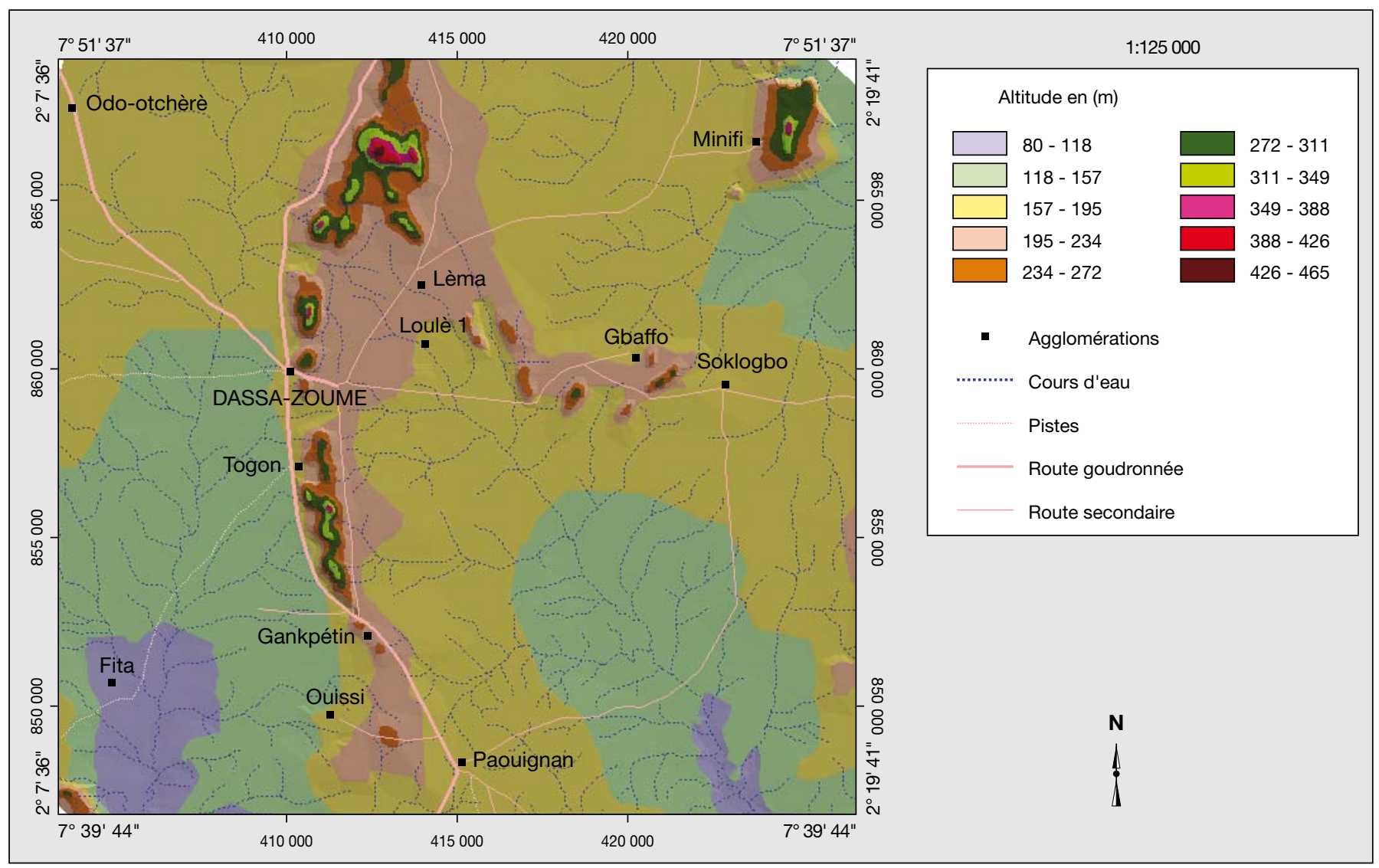

Figure 3. Modèle numérique d'altitude du secteur d'étude.

Figure 3. Digital Elevation Model of the study area. 
occidentale française, la feuille de Zagnanado à l'échelle de 1/200 000, et la carte générale du Benin au 1/600 000 (IGN France, 1967 ; IGN France et IGN Benin, 1992).

L'approche repose essentiellement sur l'exploitation de l'image Landsat de 2000 et sur l'utilisation de la carte topographique. Les données GPS ont permis de valider les résultats.

\section{Traitement et analyse des données}

Amélioration des paramètres radiométriques de l'image Landsat ETM

Dans le but de réduire les interférences dues aux conditions atmosphériques, l'image Landsat a subi un certain nombre de traitements, notamment, la réduction des effets dus au brouillard et aux bruits provenant des particules qui peuvent engendrer des réflectances exoatmosphériques. Les algorithmes Haze reduction et Noise reduction de la fonc- tion d'amélioration des comptes radiométriques du logiciel Erdas Imagine 9.1, ont été utilisés à cet effet.

\section{Détermination de l'indice \\ normalisé de végétation (NDVI) et calcul des pentes}

L'identification des bas-fonds repose sur le calcul du NDVI (normalised difference vegetation index) et sur l'utilisation de la carte de pente générée à partir du modèle numérique de terrain (MNT).

Le NDVI est determiné à partir de l'image Landsat du 13 décembre 2000, qui est une image prise en début de saison sèche, période où la biomasse végétale est généralement faible. Compte tenu de la pluviométrie de l'année 2000 qui a présenté une faible hauteur pluviométrique de $889,6 \mathrm{~mm}$, dont $0 \mathrm{~mm}$ pour les mois de novembre et de décembre, les valeurs de l'indice normalisé de végétation ont été généralement faibles. Ces faibles valeurs sont d'une importance capitale dans la localisation des écosystèmes de bas-fonds qui sont généralement des zones humides. Ainsi, les valeurs seuils sont comprises entre les valeurs - 3 et - 0,4 qui caractérisent les écosystèmes de bas-fonds.

Le calcul du NDVI a montré une forte réflectance dans le canal du proche infrarouge (PIR) le long des cours d'eau et sur les rochers contenant des fractures dans lesquelles s'est installée une végétation saxicole (figure 2). L'extraction des zones humides a pris en compte le néocanal NDVI généré, les cours d'eau, les ruisseaux et la topographie du milieu fournie par la carte de pente réalisée sur la base du MNT.

Le NDVI est un indice de végétation qui repose sur une combinaison des réflectances mesurées dans le rouge (RED) et le proche infrarouge (PIR), (Tucker 1979). Permettant de résumer en une seule variable la quantité et la vigueur de la végétation, le NDVI est calculé selon la formule ci-après :

$$
\mathrm{NDVI}=(\mathrm{PIR}-\mathrm{R}) /(\mathrm{PIR}+\mathrm{R})
$$

où le PIR correspond à la bande du proche infrarouge et $\mathrm{R}$ celle du rouge (Turckey, 1979; Hiroyuki, 2005).

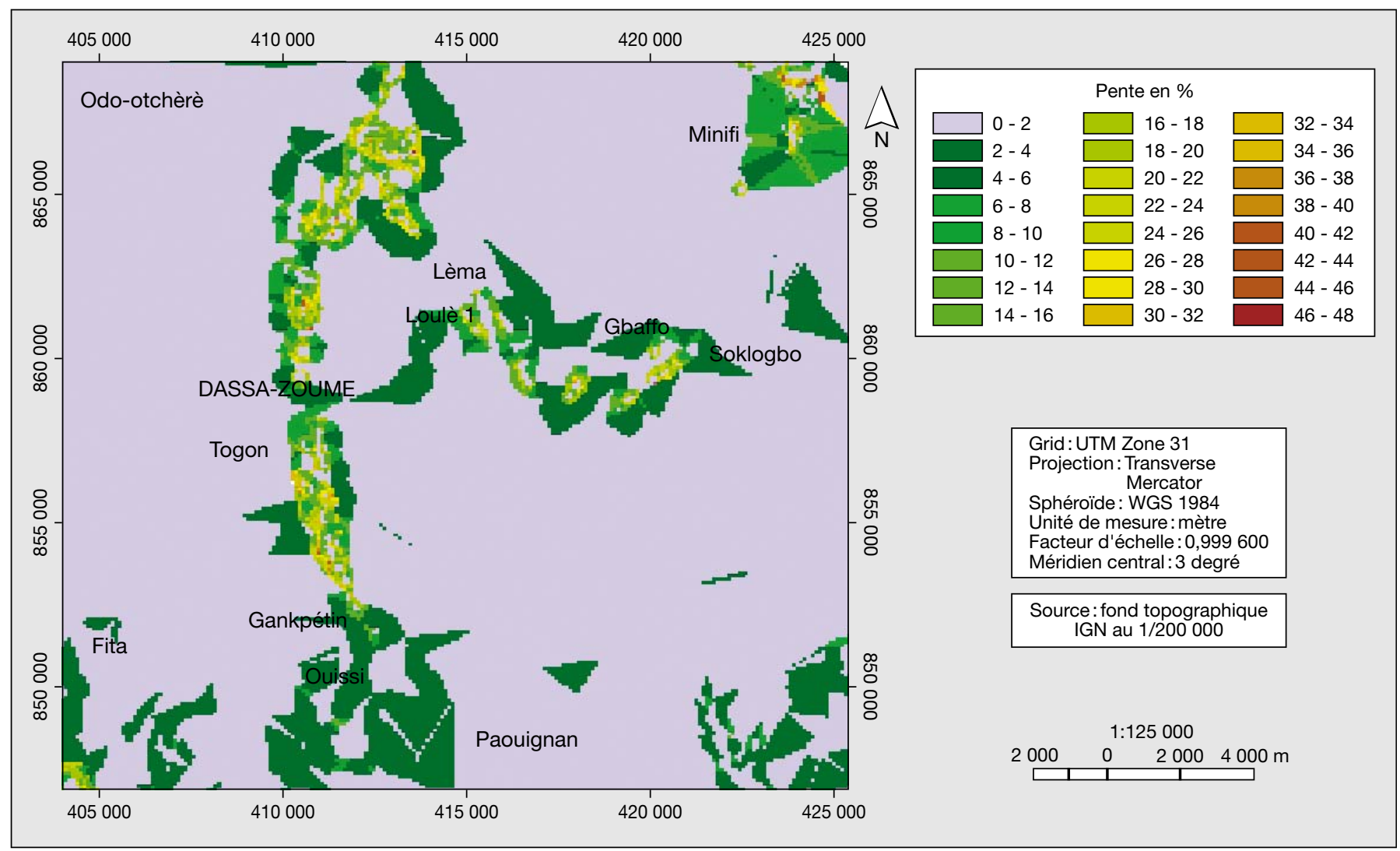

Figure 4. Carte des pentes.

Figure 4. Slope map. 
La forte réflectance exprimée en bleu au niveau des zones humides et des rochers traduit la forte capacité en biomasse de ces zones et le degré important d'humidité du sol.

La sélection des zones humides dans lesquelles sont intégrés les bas-fonds a été faite par la superposition des pentes fortes sur le néocanal NDVI. Cette carte de pente a été vectorisée en vue de faciliter la superposition. Cette superposition est accompagnée de celle des cours d'eau et ruisseaux du secteur d'étude et a permis d'éliminer les zones à forte capacité en biomasse dans lesquelles se trouvent les pentes fortes, rocheuses, et d'identifier les zones humides basses.

Les zones de bas-fonds ont été ensuite extraites en reprenant le concept de bas-fonds tel que défini dans le DIARPA (diagnostic rapide de pré-aménagement [Legoupil et al., 2000 ; Jamin et al., 2002]), à savoir des zones dépressionnaires situées en amont du réseau hydrographique et dont la superficie est limitée (moins de 25 hectares).
Une telle sélection est possible par la fonction SIG posant comme condition de l'attribut : superficie des zones humides inférieure ou égale à 25 ba.

\section{Réalisation du MNT et modèle en 3D}

Les courbes de niveau ont été numérisées puis exportées dans l'environnement du logiciel ArcGIS 9.2, pour la génération du modèle d'élévation de terrain et le calcul des pentes. La carte de pente obtenue a été classifiée. Cette classification a été faite en tenant compte des contraintes précisées par le DIARPA (Legoupil et al., 2000 ; Jamin et al., 2002), qui considère l'aménagement comme techniquement et économiquement intéressant si les pentes sont inférieures ou égales à $2 \%$.

\section{Méthode de validation des résultats}

Dans le but de vérifier l'effectivité des bas-fonds inventoriés à travers l'approche proposée par l'étude, un échantillon de bas-fonds a été sélectionné et vérifié sur le terrain. La méthode par quota a été utilisée avec un taux d'échantillonnage de $8 \%$ sur le nombre total de bas-fonds inventoriés. La superposition des points GPS de ces bas-fonds échantillonnés sur la carte des bas-fonds inventoriés, a permis de valider les résultats obtenus et de conclure à l'efficacité de l'approche adoptée.

\section{Résultats et discussion}

\section{Génération d'un MNT et analyse du terrain en 3 dimensions}

Le MNT obtenu (figure 3) montre les différentes facettes topographiques où les cours d'eau prennent naissance. Ces zones constituent la partie amont du

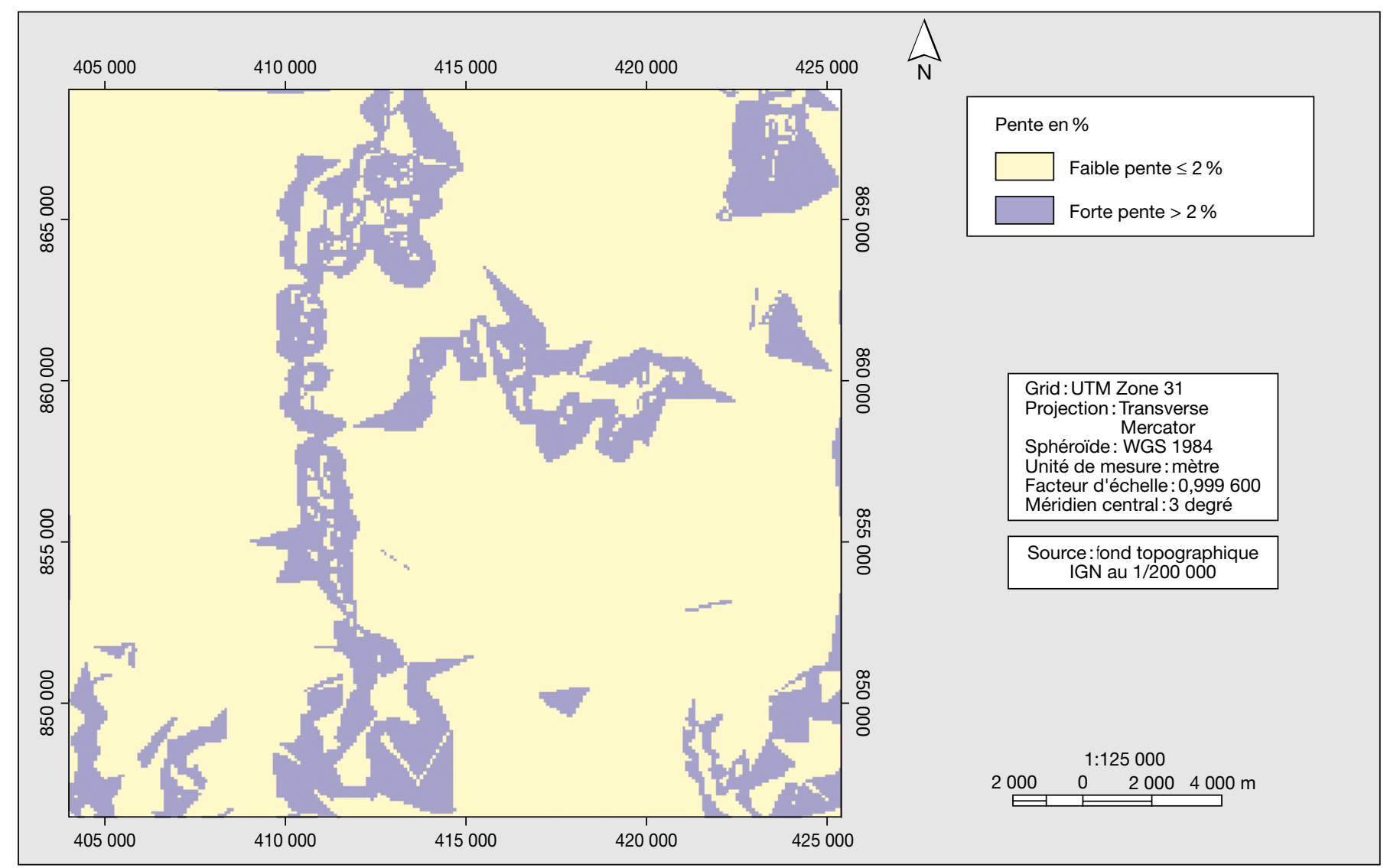

Figure 5. Carte des classes de pente.

Figure 5. Map of slope classes. 
réseau hydrographique, où les processus de sédimentation alluviale sont d'importance mineure. Le MNT montre que la commune de Dassa-Zoumé se situe sur la ligne de partage des eaux entre les cours d'eau Olodjo et Têwi.

\section{Estimation des pentes}

La carte de pente obtenue (figure 4) montre que, dans la zone d'étude, les pentes varient de 0 à plus de $48 \%$. Ces valeurs montrent que la zone d'étude est par endroits très accidentée. La répartition de ces pentes en neuf classes a permis de mieux cerner les zones de bas-fonds probables. Compte tenu de la limite de $2 \%$ de pente que nous avons retenue, nous avons distingué les pentes faibles (inférieures ou égales à $2 \%$ ) et les pentes fortes (supérieures à $2 \%$ ), non facilement aménageables (figure 5).

\section{Potentialités en bas-fonds de la zone d'étude}

L'extraction des bas-fonds s'effectue à partir d'une requête spatiale retenant les zones humides ayant une superficie inférieure ou égale à 25 hectares.

La figure 6 montre que 181 bas-fonds ont été identifiés dans la zone d'étude, pour une superficie totale de 1124 hectares.

\section{Validation de l'approche d'inventaire proposée}

La quinzaine de bas-fonds échantillonnés sur les 181 inventoriés (environ 8\%), a permis de tester l'efficacité de l'approche utilisée dans le cadre de cette étude. Ainsi la vérification de l'effectivité de ces basfonds sur le terrain s'est montrée positive. Tous les points GPS relevés dans les bas-fonds sur le terrain ont été facilement superposés sur la carte des bas-fonds inventoriés et confirment les critères de choix à savoir les seuils de - 0,4 à - 0,3 pour le NDVI et une pente inférieure ou égale à $2 \%$ (figure 7 ).

\section{Discussion}

L'approche par télédétection a permis d'inventorier les différents bas-fonds du secteur étudié. Cette approche se révèle plus rapide que l'approche terrain directe de caractérisation des agro-écosystèmes de bas-fonds, qui nécessite plus de mobilisation de ressources matérielles et humaines (Andriesse et al., 1994).

La faible résolution de l'image $(28,5 \mathrm{~m})$ ne permet pas de réaliser la cartographie individuelle des bas-fonds, mais elle permet d'identifier et de répertorier tous les bas-fonds aménageables de façon exhaustive.

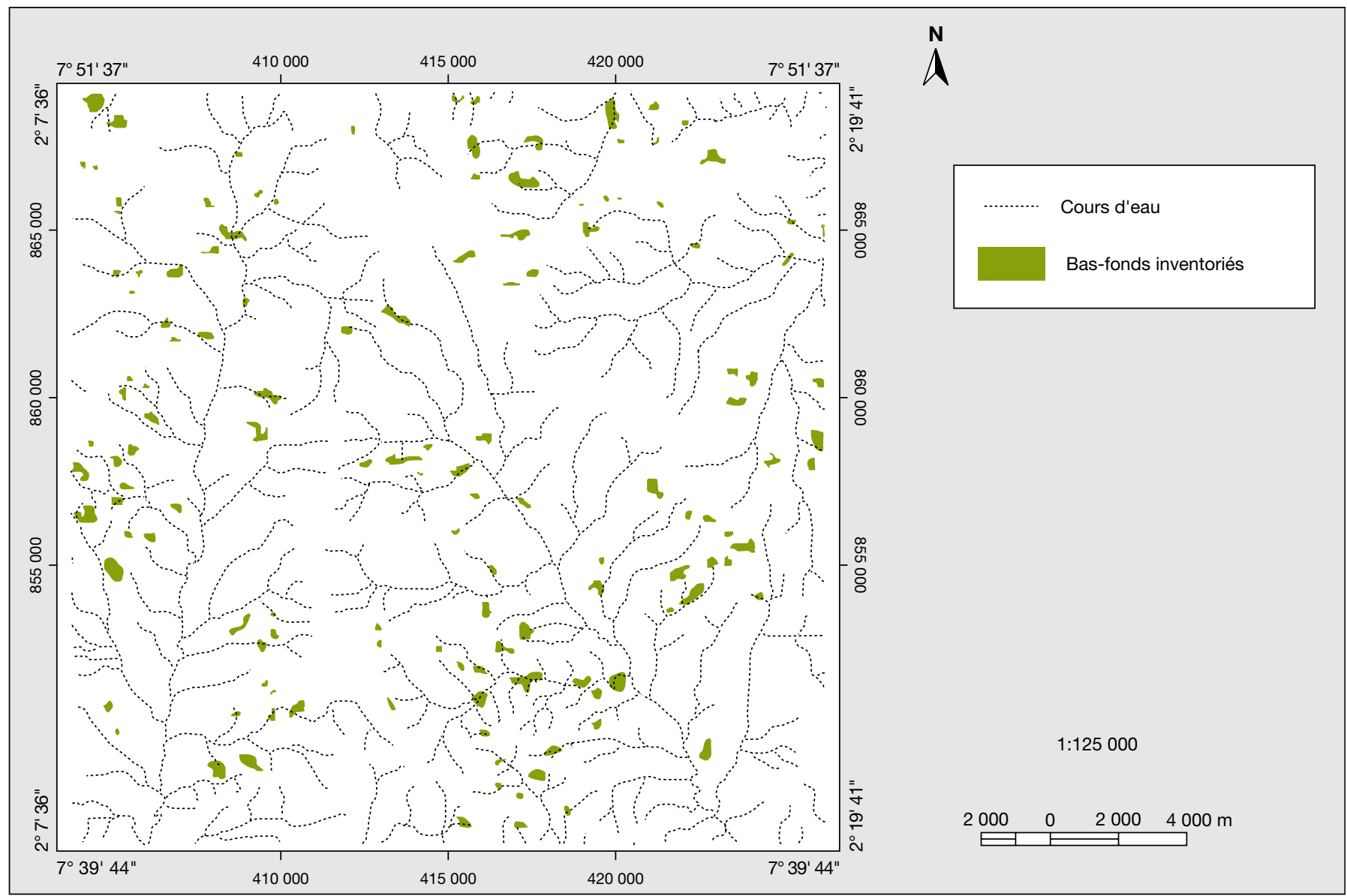

Figure 6. Les bas-fonds du secteur d'étude.

Figure 6. Inland valleys in the study areas. 


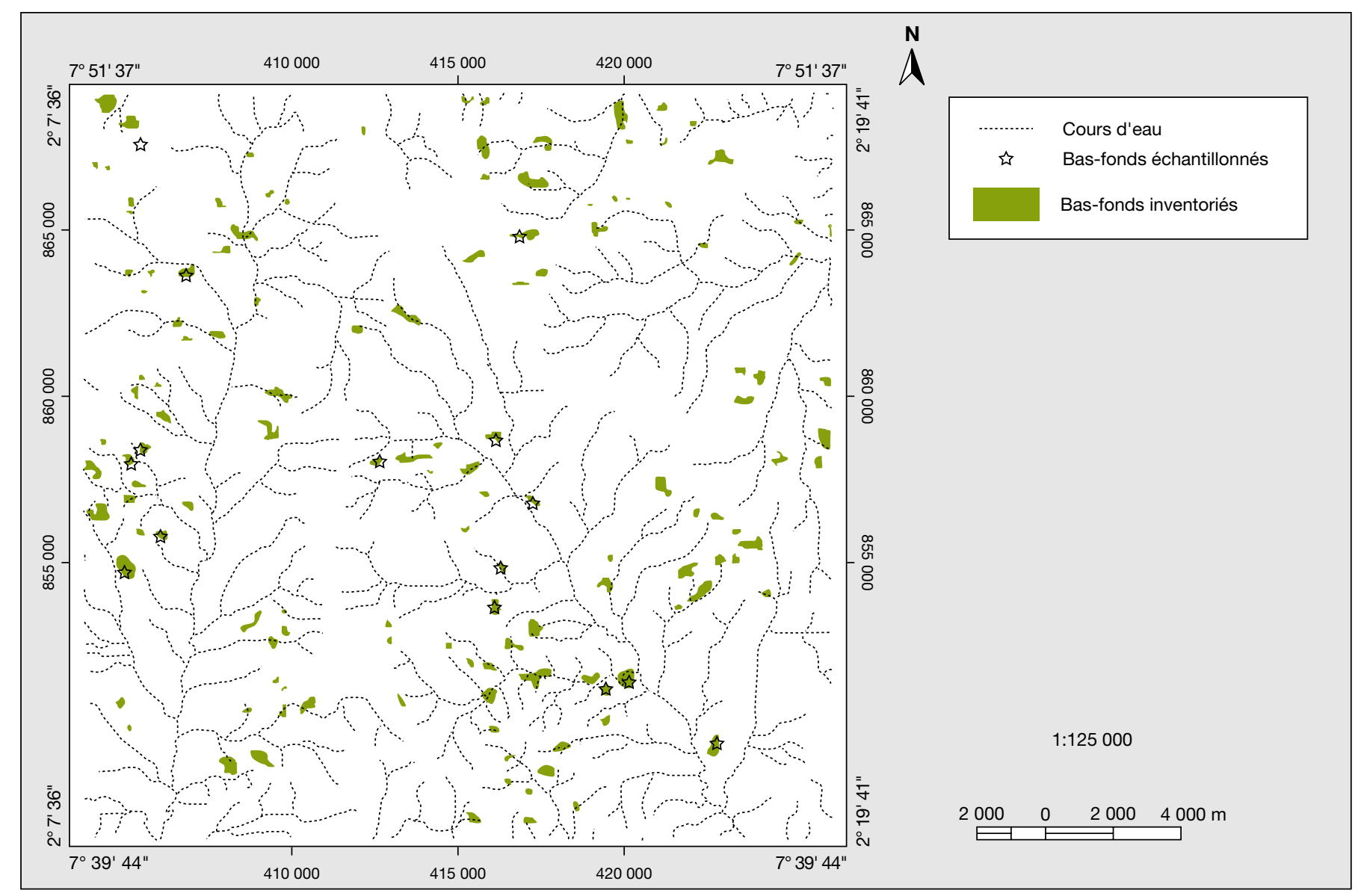

Figure 7. Localisation des bas-fonds échantillonnés pour la validation des résultats.

Figure 7. Location of sampled inland valleys for the validation of results.

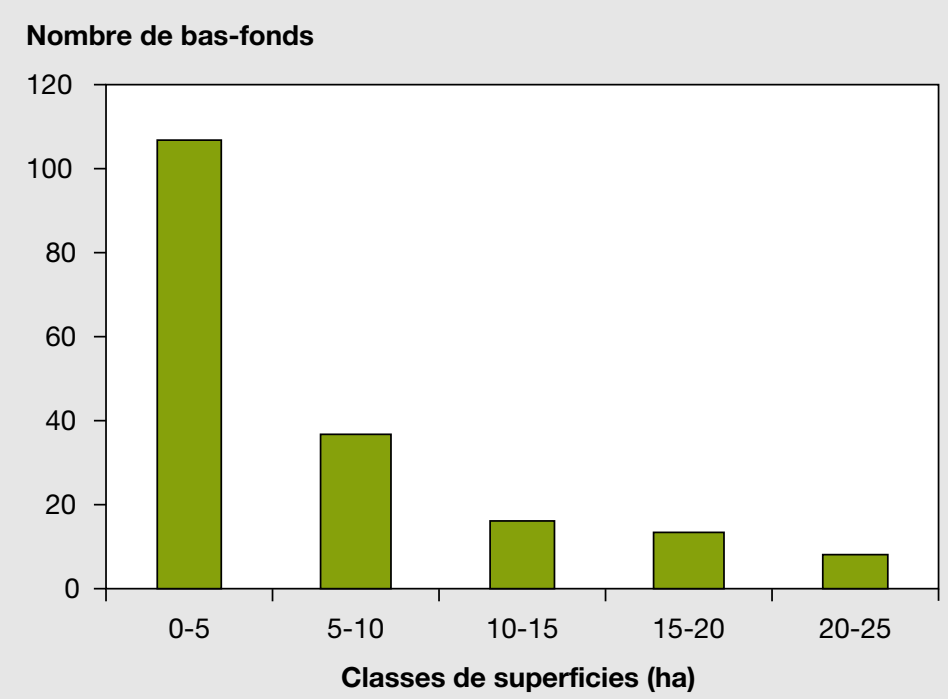

Figure 8. Superficies des bas-fonds.

Figure 8. Areas of the inland valleys.
La plus petite taille de bas-fond obtenue par cette méthode est de 0,21 hectare, soit 2,5 fois la taille d'un pixel. Sur les 181 basfonds dénombrés, 107 ont des superficies comprises entre 0 et 5 hectares, soit $60 \%$ des bas-fonds de la zone (figure 8). Ces bas-fonds identifiés peuvent déjà faire l'objet d'une mise en valeur car ils présentent des caractéristiques hydrologiques et morphologiques qui correspondent aux critères de mise en valeur proposés par le DIARPA (Windmeijer et al., 2002).

\section{Conclusion}

La méthode a permis la détection des basfonds à partir du néocanal NDVI, ainsi que la distinction de ceux qui sont aménageables sur la base d'un critère de pente inférieure à $2 \%$. L'approche de 
détection et d'inventaire utilisée dans cette étude, essentiellement fondée sur l'exploitation d'une image satellitaire, présente une fiabilité telle qu'elle mérite d'être généralisée dans la recherche des zones basses pour des perspectives agricoles. Cette approche est simple, plus rapide et moins onéreuse en ce sens qu'elle permet de relever en un temps record le maximum de bas-fonds et de réduire le coût des phases de terrain de l'inventaire des bas-fonds d'une zone donnée. Dans la même logique de comparaison par rapport aux autres approches, notamment l'approche de caractérisation détaillée proposées dans les pays africains participant au Consortium Bas-fonds (CBF-IVC), la présente approche permet de gagner en temps et de réduire l'intensivité et l'ampleur des travaux de terrain préalables. Toutefois, bien que cette approche soit simple et moins coûteuse, elle nécessite des travaux complémentaires afin de cerner les caractéristiques socio-économiques et biophysiques des bas-fonds inventoriés. Par ailleurs, l'utilisation systématique de notre méthode par les projets de développement agricole des bas-fonds permettrait la mise en place progressive d'un système d'information sur les bas-fonds aux niveaux national et régional du consortium CBF-IVC.

\section{Références}

Afouda F. L'eau et les cultures dans le Bénin central et septentrional: étude de la variabilité des bilans de l'eau dans leurs relations avec le milieu rural de la savane africaine. Thèse de doctorat, iniversité de Paris IV-Sorbone, Paris, 1990.

Andriesse W, Fresco LO, van Duivenbooden N Windmeijer PN. Multi-scale Characterization of Inland Valley Agro-ecosystems in West Africa. Netherlands Journal of Agricultural Science $1994 ; 42$ : 159-79.

Andriesse W, Fresco LO. A Characterization of Rice-growing Environments in West Africa. Agric Ecosys and Environ 1991 ; 33 : 377-95.

Danvi C, Mama VJ. État de l'art de valorisation des bas-fonds au Bénin. Rapport de projet. Cotonou : Unité nationale de Coordination du Consortium Bas-fond, 1998.

Hiroyuki Y. Observation of riparian vegetation in western namibia by using ndvi and ndwi derived from spot-vegetation. African Study Monographs 2005 ; (Suppl.30) : 153-63.

Houndagba CJ, Akoègninou A. Contraintes écologiques et mise en valeur des bas-fonds dans la région de Dassa-Zoumé (centre Bénin). J Rech Sci Univ Bénin (Togo) 1999 ; 3 : 30-9.

IGN Paris (Service géographique à Dakar). Carte de l'Afrique de l'Ouest. Feuille topographique de Zagnanado au 1/200 000. $1^{\text {ere }}$ édition: Décembre 1960 ; République du Dahomey, Feuille NB-31$\mathrm{XXI}$

IGN Paris et IGN Cotonou. République du Bénin. Carte générale à $1 / 600000.2^{\mathrm{e}}$ édition. Paris Cotonou : IGN, 2000.

INSAE. Troisième recensement général de la population et de l'habitation. Février 2002. Quelques résultats. Cotonou: INSAE, 2003.
Jamin JY, Biaou F Legoupil JC Lidon B. Le diagnostic rapide de pré-aménagement (DIARPA): un outil d'aide à la décision pour les aménagements de bas-fonds. In: Mémento de l'agronome. Paris; Montpellier: Gret; Cirad, 2002 (cédérom).

Legoupil JC, Lidon B, Blanchet F, Jamin JY. Mise en valeur et aménagement des bas-fonds d'Afrique de l'Ouest: proposition d'un outil d'aide à l'aménagement, le diagnostic rapide de pré-aménagement (DIARPA). Synthèse des résultats du Cirad et de ses partenaires sur la caractérisation des bas-fonds et l'intensification de leur mise en valeur. Bouaké, 2000 (cédérom).

Mama VJ, Orekan V, Agli C, et al. Développement participatif des technologies rizicoles dans les bas-fonds de Gankpétin et de Gomé (Centre Bénin). Bulletin de la Recherche Agronomique du Bénin 2000 ; (29) : 1-15.

Maméri C, Mohamed K, Miézan MK. Intensification de la riziculture de bas-fonds dans le SineSaloum (Sénégal). Cah Agric 2008; 17: 4515.doi : 10.1684/agr.2008.0205

Raunet M. Bas-fonds et riziculture en Afrique; approche structurale et comparative. Agronomie Tropicale $1985 ; 40: 181-201$.

Tucker CJ. Red and photographic infrared linear combinations for monitoring vegetation. Remote Sensing of Environment 1979 ; 8 : 127-50.

Windmeijer PN, Dugué MJ, Jamin JY, van de Giesen N. Describing hydrological characteristics for inland valley development. Proceedings of the Second Scientific Workshop of the Inland Valley Consortium/Présentation des caractéristiques hydrologiques de la mise en valeur des bas-fonds. Compte rendu du Deuxième Atelier Scientifique du Consortium Bas-fonds, Bouaké, 2002. 